International Mathematical Forum, 2, 2007, no. 47, 2305 - 2319

\title{
Linear Preservers of Idempotence on Triangular Matrix Spaces over any Field
}

\author{
Jin-Li Xu, Chong-Guang Cao and Xiao-Min Tang \\ Department of Mathematics \\ Heilongjiang University \\ Harbin, 150080, P. R. China \\ jclixv@163.com (J.L. Xu), x.m.tang@163.com (X.M. Tang)
}

\begin{abstract}
Let $\mathbf{F}$ be any field and let $T_{n}(\mathbf{F})$ be the $n \times n$ upper triangular matrix space over $\mathbf{F}$. We denote the set of all $n \times n$ upper triangular idempotent matrices over $\mathbf{F}$ by $P_{n}(\mathbf{F})$. A map $\varphi$ on $T_{n}(\mathbf{F})$ is called a preserver of idempotence if $\varphi\left(P_{n}(\mathbf{F})\right) \subset P_{n}(\mathbf{F})$; and a strong preserver of idempotence if $\varphi\left(P_{n}(\mathbf{F})\right)=P_{n}(\mathbf{F})$. In this paper, we characterize the bijective linear preservers of idempotence on $T_{n}(\mathbf{F})$. Further, the strong linear preservers of idempotence on $T_{n}(\mathbf{F})$ are characterized.
\end{abstract}

Mathematics Subject Classifications: 15A04; 15A03

Keywords: Field; triangular matrix; idempotence

\section{Introduction and main result}

In the past six decades, many authors studied linear preserver problems (see[115]) which are concerning with characterizing maps on matrix spaces that preserve some property, set or relation. As pointed out in $[5,7]$, one of techniques that have been successful in solving linear preserver problems is to reducing a new problem to a known one, while the latter is often a preserver of idempotence. The preservers of idempotence play an important role in studying 
linear preserver problems, and thus there are a lot of results about the linear preserver of idempotence $[1-4,8,10-12]$. Expressly, the linear preservers of idempotence on triangular matrices are discussed in $[4,10-12]$. These papers obtained the forms of linear preserver of idempotence on triangular matrices with a common restriction, that is, they require the field is of characteristic not 2 (or 2 is a unit of the ring). There is a natural problem, if the field is of characteristic 2, how determine the forms of linear preserver of idempotence on triangular matrices? In this paper, we will answer this question. We solve this problem over any field including the field of characteristic 2. As we will see, our results contain some new forms.

We now introduce some concepts and fix the notation, which will be used in the rest of this article. Suppose $\mathbf{F}$ is a field and $\mathbf{F}^{*}$ is its subset consisting of all nonzero elements, Char $\mathbf{F}$ denotes the characteristic of $\mathbf{F}$. For any positive integer $n$, let $T_{n}(\mathbf{F})$ be the linear space of all $n \times n$ upper triangular matrices over $\mathbf{F}$ and denote the set of all upper triangular idempotent matrices by $P_{n}(\mathbf{F})$, namely, $P_{n}(\mathbf{F})=\left\{A \in T_{n}(\mathbf{F}): A^{2}=A\right\}$. For any matrix $B \in T_{n}(\mathbf{F})$, $B^{T}$ (respectively, $\operatorname{rank} B$ ) denotes the transpose (respectively, rank) of $B$. Let $I_{n}$ be the $n \times n$ identity matrix. For any positive integers $i$ and $j$, let $E_{i j}$ be the matrix with 1 in the $(i, j)$-th entry and 0 elsewhere. For a subset $S$ of $T_{n}(\mathbf{F})$, let $\operatorname{span}(S)$ be the space spanned by $S$. Symbol $\oplus$ denotes the usual direct sum of matrices. For two integers $i<j$, we let $[i, j]=\{i, i+1, \cdots, j\}$.

A Linear map $\varphi$ from $T_{n}(\mathbf{F})$ to itself is called linear preserver of idempotence if $\varphi\left(P_{n}(\mathbf{F})\right) \subset P_{n}(\mathbf{F})$; and a strong linear preserver of idempotence if $\varphi\left(P_{n}(\mathbf{F})\right)=P_{n}(\mathbf{F})$.

We state our main results as follows.

Theorem 1.1 Suppose $\mathbf{F}$ is any field and $n \geq 1$ is an integer. Then $\varphi$ is a bijective linear preserver of idempotence on $T_{n}(\mathbf{F})$ if and only if there is an invertible matrix $T \in T_{n}(\mathbf{F})$ such that

$$
\varphi(A)=T A T^{-1}+f(A) I_{n} \text { for all } A \in T_{n}(\mathbf{F})
$$

or

$$
\varphi(A)=T J A^{T} J T^{-1}+f(A) I_{n} \text { for all } A \in T_{n}(\mathbf{F}),
$$


where $J=\sum_{k=1}^{n} E_{k, n-k+1}$ and $f$ is a linear map from $T_{n}(\mathbf{F})$ to $\mathbf{F}$ satisfying (a) $f\left(I_{n}\right)=0 ;(b) f\left(E_{k k}\right) \in\{0,1\}$ for all $k \in[1, n]$; (c) if char $\mathbf{F} \neq 2$ then $f=0 ;(d)$ if $\mathbf{F} \neq \mathbf{F}_{2}$, then $f\left(E_{i j}\right)=0$ for all $i<j$.

Theorem 1.2 Suppose $\mathbf{F}$ is any field and $n \geq 1$ is an integer. Then $\varphi$ is a strong linear preserver of idempotence on $T_{n}(\mathbf{F})$ if and only if $\varphi$ is of the forms (1) $\operatorname{or}(2)$.

About Theorem 1.1, we give a remark as follows.

Remark 1.3 Let $\varphi$ be a map on $T_{n}(\mathbf{F})$ defined by $\varphi(A)=a_{11} I_{n}$ for all $A=$ $\left[a_{i j}\right] \in T_{n}(\mathbf{F})$. Then $\varphi$ is a linear preserver of idempotence on $T_{n}(\mathbf{F})$ but it is not of the form which given by Theorem 1.1. This show that the assumption of bijection of $\varphi$ in Theorem 1.1 is essential.

In fact, if we take out the assumption of bijection of $\varphi$, then the forms of such maps become very complicated. For example, the following $\varphi$ give a lot of linear preservers of idempotence on $T_{n}(\mathbf{F})$ which are different from the forms (1) or (2).

Example 1.4 Let $\varphi$ be a map on $T_{n}(\mathbf{F})$ defined by

$$
\varphi(A)=\left(A_{1} \otimes B_{1}\right) \oplus \cdots \oplus\left(A_{t} \otimes B_{t}\right) \text { for all } A \in T_{n}(\mathbf{F})
$$

where $A_{i}, i=1, \cdots t$ are some $s_{i} \times s_{i}$ special principal submatrices of $A$ such that

$$
A=\left[\begin{array}{cccc}
A_{1} & * & * & * \\
0 & \ddots & * & * \\
0 & 0 & A_{t} & * \\
0 & 0 & 0 & *
\end{array}\right]
$$

and $B_{i}, i=1, \cdots t$ are respectively some given $m_{i} \times m_{i}$ idempotent upper triangular matrices satisfying $\sum_{i=1}^{t} s_{i} m_{i}=n$. Then it is easy to check that $\varphi$ is a linear preserver of idempotence. 


\section{Preliminary results}

For the proof our main results we need several lemmas.

Lemma 2.1 Suppose that $A, B, A+B \in P_{n}(\mathbf{F})$. Then $A B=B A$. We have in addition $A B=0$ if $\operatorname{Char} \mathbf{F} \neq 2$.

Proof. The proof is simple and we omitted it.

Lemma 2.2 [6] Suppose $A \in P_{n}(\mathbf{F})$, then there is an invertible matrix $T \in$ $T_{n}(\mathbf{F})$ such that

$$
A=T \operatorname{diag}\left(\varepsilon_{1}, \varepsilon_{2}, \cdots, \varepsilon_{n}\right) T^{-1}
$$

where $\varepsilon_{1}, \varepsilon_{2}, \cdots, \varepsilon_{n} \in\{0,1\}$.

Lemma 2.3 Suppose $\mathbf{F}$ is any field and $n \geq 1$ is an integer. Suppose

$A_{1}, A_{2}, \cdots, A_{m} \in T_{n}(\mathbf{F})$ are idempotent matrices such that $A_{i} A_{j}=A_{j} A_{i}$ for all $i, j \in[1, m]$. Then there are an invertible matrix $T \in T_{n}(F)$ and $\varepsilon_{i j} \in$ $\{0,1\}, i=1,2, \cdots, n, \quad j=1,2, \cdots$, m such that

$$
A_{k}=T \operatorname{diag}\left(\varepsilon_{1 k}, \varepsilon_{2 k}, \cdots, \varepsilon_{n k}\right) T^{-1} \text { for all } k \in[1, m]
$$

Proof. We prove the result by induction on $m$.

Due to Lemma 2.2, the conclusion is true for $m=1$. Suppose the conclusion is true for $m-1$, that is there exist an invertible matrix $T_{1} \in T_{n}(F)$ and $\varepsilon_{i j} \in\{0,1\}, i=1,2, \cdots, n, \quad j=1,2, \cdots, m-1$ such that

$$
A_{k}=T_{1} \operatorname{diag}\left(\varepsilon_{1 k}, \varepsilon_{2 k}, \cdots, \varepsilon_{n k}\right) T_{1}^{-1} \text {, for all } k \in[1, m-1] \text {. }
$$

We will show that the conclusion is true for $m$, too. Let $A_{m}=T_{1}\left[a_{i j}\right] T_{1}^{-1}$. So $\left[a_{i j}\right] \in P_{n}(\mathbf{F})$ since $A_{m} \in P_{n}(\mathbf{F})$. By the situation of the nonzero elements in $\left[a_{i j}\right]$, we can find a $n \times n$ permutation matrix $P$ such that

$$
A_{m}=T_{1} P\left(B_{1} \oplus B_{2} \oplus \cdots \oplus B_{t} \oplus 0\right) P^{-1} T_{1}^{-1}
$$

here $B_{i} \in T_{s_{i}}(\mathbf{F}), i=1, \cdots, t$ are indecomposable idempotent submatrices of $\left[a_{i j}\right]$ (recall that an upper triangular matrix $N$ is called indecomposable if there is not any permutation matrix $Q$ such that $Q N Q^{-1}$ decomposes into a 
nontrivial direct sum of upper triangular matrices), and $s_{1}, \cdots, s_{t}$ are some positive integers.

For every $k \in[1, m-1]$, it follows form $A_{k} A_{m}=A_{m} A_{k}$ that

$$
a_{i j}\left(\varepsilon_{i k}-\varepsilon_{j k}\right)=0 \text { for all } k \in[1, m-1] .
$$

Indeed, $\varepsilon_{i k}=\varepsilon_{j k}$ if $a_{i j} \neq 0$ for all $k \in[1, m-1]$. This, together with (3), one can determine $t$ non-intersectant subsets of $[1, n]$, say $\left\{q_{11}, q_{12}, \cdots, q_{1 s_{1}}\right\}$, $\left\{q_{21}, q_{22}, \cdots, q_{2 s_{2}}\right\}, \cdots,\left\{q_{t 1}, q_{t 2}, \cdots, q_{t s_{t}}\right\}$, such that for every $i \in[1, t]$,

$$
\varepsilon_{q_{i 1} k}=\varepsilon_{q_{i 2} k}=\cdots=\varepsilon_{q_{i_{i}} k} \in\{0,1\} \text { for all } k \in[1, m-1] .
$$

Thus, by (4) and the choice of $P$, one has

$$
A_{k}=T_{1} P\left(C_{k 1} \oplus C_{k 2} \oplus \cdots \oplus C_{k t} \oplus C_{k}\right) P^{-1} T_{1}^{-1} \text { for all } k \in[1, m-1]
$$

where $C_{k i}=I_{s_{i}}$ or $0_{s_{i}}, i=1, \cdots, t$ and $C_{k}$ is a diagonal matrix with diagonal elements 0 or 1 .

Now, note that $B_{1}, B_{2}, \cdots, B_{t}$ are idempotent upper triangular matrices in (3). Due to Lemma 2.2, then there are invertible upper triangular matrices $U_{1}, \cdots, U_{t}$ such that

$$
B_{i}=U_{i} \operatorname{diag}\left(b_{i 1}, \cdots, b_{i s_{i}}\right) U_{i}^{-1} \quad \text { for all } i \in[1, t],
$$

here $b_{i j} \in\{0,1\}$. Put $U=U_{1} \oplus U_{2} \oplus \cdots \oplus U_{t} \oplus I$, then $U$ is invertible in $T_{n}(\mathbf{F})$. We have by (5) and (3) that

$$
A_{k}=T_{1} P U\left(C_{k 1} \oplus C_{k 2} \oplus \cdots \oplus C_{k t} \oplus C_{k}\right) U^{-1} P^{-1} T_{1}^{-1} \text { for all } k \in[1, m-1]
$$

and

$$
A_{m}=T_{1} P U\left(\left[\begin{array}{ccc}
b_{11} & & \\
& \ddots & \\
& & b_{1 s_{1}}
\end{array}\right] \oplus \cdots \oplus\left[\begin{array}{ccc}
b_{t 1} & & \\
& \ddots & \\
& & b_{t s t}
\end{array}\right] \oplus 0\right) U^{-1} P^{-1} T_{1}^{-1} .
$$

Let $T=T_{1} P U P^{-1}$. By the choice of $P$ and $U$, it is not difficult to see that $P U P^{-1}$ is an upper triangular matrix. Hence, $T \in T_{n}(\mathbf{F})$. Finally, by (6) and (7) we see that $T^{-1} A_{k} T, k=1,2, \cdots, m$ are diagonal matrices with diagonal elements 0 or 1 . The proof is completed. 
Lemma 2.4 For a given subset $\Lambda$ of $[1, n]$ with $r$ elements, put

$$
\Gamma_{n}(\Lambda)=\left\{A \in T_{n}(\mathbf{F}): A\left(\sum_{j \in \Lambda} E_{j j}\right)=\left(\sum_{j \in \Lambda} E_{j j}\right) A\right\} .
$$

Then $\operatorname{dim} \operatorname{span}\left(\Gamma_{n}(\Lambda)\right) \leq 2^{-1}\left(n^{2}-2 n r+n+2 r^{2}\right)$.

Proof. We prove the result by induction on $n$. The case $n=1$ is clear. Suppose the conclusion is true for $n-1$. For any $A \in \Gamma_{n}(\Lambda)$, we write $A$ as

$$
A=\left[\begin{array}{cc}
A_{n-1} & \delta \\
0 & a_{n n}
\end{array}\right]
$$

where $a_{n n} \in \mathbf{F}, A_{n-1} \in T_{n}(\mathbf{F})$. Clearly, $A_{n-1} \in \Gamma_{n-1}\left(\Lambda_{1}\right)$ where $\Lambda_{1}=\Lambda \backslash\{n\}$.

Case 1. $n \in \Lambda$. By $A\left(\sum_{j \in \Lambda} E_{j j}\right)=\left(\sum_{j \in \Lambda} E_{j j}\right) A$, we see that $\left(\sum_{j \in \Lambda} E_{j j}\right)\left[\begin{array}{c}\delta \\ a_{n n}\end{array}\right]=\left[\begin{array}{c}\delta \\ a_{n n}\end{array}\right]$. Hence, $\left[\begin{array}{c}\delta \\ a_{n n}\end{array}\right]$ contains at most $r$ nonzero elements. Thus, by induction hypothesis we have

$$
\begin{aligned}
& \operatorname{dim} \operatorname{span}\left(\Gamma_{n}(\Lambda)\right) \leq \operatorname{dim} \operatorname{span}\left(\Gamma_{n-1}\left(\Lambda_{1}\right)\right)+r \\
& \leq 2^{-1}\left((n-1)^{2}-2(n-1)(r-1)+(n-1)+2(r-1)^{2}\right)+r \\
& =2^{-1}\left(n^{2}-2 n r+n+2 r^{2}\right) .
\end{aligned}
$$

Case 2. $n \notin \Lambda$. By $A\left(\sum_{j \in \Lambda} E_{j j}\right)=\left(\sum_{j \in \Lambda} E_{j j}\right) A$, we see that $\left(\sum_{j \in \Lambda} E_{j j}\right)\left[\begin{array}{c}\delta \\ a_{n n}\end{array}\right]=0$. Thus, $\left[\begin{array}{c}\delta \\ a_{n n}\end{array}\right]$ contains at most $n-r$ nonzero elements. It follows from the induction hypothesis that

$$
\begin{aligned}
& \operatorname{dim} \operatorname{span}\left(\Gamma_{n}(\Lambda)\right) \leq \operatorname{dim} \operatorname{span}\left(\Gamma_{n-1}\left(\Lambda_{1}\right)\right)+n-r \\
& \leq 2^{-1}\left((n-1)^{2}-2(n-1) r+(n-1)+2 r^{2}\right)+n-r \\
& =2^{-1}\left(n^{2}-2 n r+n+2 r^{2}\right) .
\end{aligned}
$$

We complete the proof.

\section{$3 \quad$ The proof of main results}

In this section, we assume that $\mathbf{F}$ is any field and $n \geq 1$ is an integer. 
Lemma 3.1 Let $\varphi$ be a bijective linear preserver of idempotence on $T_{n}(\mathbf{F})$. Then for any $k \in[1, n]$, we have rank $\varphi\left(E_{k k}\right)=1$ or $n-1$.

Proof. Case 1. char $\mathbf{F} \neq 2$. For any $i \neq j$, since $E_{i i}, E_{j j}, E_{i i}+E_{j j} \in P_{n}(\mathbf{F})$, we have by Lemmas 2.1 and 2.3 that $\varphi\left(E_{i i}\right) \varphi\left(E_{j j}\right)=0$ and $\varphi\left(E_{11}\right), \varphi\left(E_{22}\right), \cdots, \varphi\left(E_{n n}\right)$ can be simultaneously diagonalizable. It follows that $\operatorname{rank} \varphi\left(E_{k k}\right)=1$ for all $k \in[1, n]$.

Case 2. $\operatorname{char} \mathbf{F}=2$. It is clear that the conclusion is true when $n=1$. We assume that $n \geq 2$. For a given $k \in[1, n]$, suppose $\operatorname{rank} \varphi\left(E_{k k}\right)=r$. By $\varphi\left(E_{k k}\right) \in P_{n}(\mathbf{F})$ and Lemma 2.2, one can find an invertible matrix $T \in T_{n}(\mathbf{F})$ such that

$$
\varphi\left(E_{k k}\right)=T \operatorname{diag}\left(\varepsilon_{1}, \varepsilon_{2}, \cdots, \varepsilon_{n}\right) T^{-1}
$$

where $\varepsilon_{1}, \varepsilon_{2}, \cdots, \varepsilon_{n} \in\{0,1\}$. Take

$$
\left[\begin{array}{cc}
A_{(k-1) \times(k-1)} & B_{(k-1) \times(n-k)} \\
0 & C_{(n-k) \times(n-k)}
\end{array}\right] \in P_{n-1}(\mathbf{F}) .
$$

By Lemma 2.1 and

$$
E_{k k}+\left[\begin{array}{ccc}
A_{(k-1) \times(k-1)} & 0 & B_{(k-1) \times(n-k)} \\
0 & 0 & 0 \\
0 & 0 & C_{(n-k) \times(n-k)}
\end{array}\right] \in P_{n}(\mathbf{F})
$$

we have

$$
\begin{aligned}
& \varphi\left(E_{k k}\right) \varphi\left(\left[\begin{array}{ccc}
A_{(k-1) \times(k-1)} & 0 & B_{(k-1) \times(n-k)} \\
0 & 0 & 0 \\
0 & 0 & C_{(n-k) \times(n-k)}
\end{array}\right]\right) \\
= & \varphi\left(\left[\begin{array}{ccc}
A_{(k-1) \times(k-1)} & 0 & B_{(k-1) \times(n-k)} \\
0 & 0 & 0 \\
0 & 0 & C_{(n-k) \times(n-k)}
\end{array}\right]\right) \varphi\left(E_{k k}\right) .
\end{aligned}
$$

Let

$$
S=\left\{\left[\begin{array}{ccc}
A_{(k-1) \times(k-1)} & 0 & B_{(k-1) \times(n-k)} \\
0 & a & 0 \\
0 & 0 & C_{(n-k) \times(n-k)}
\end{array}\right]: a \in \mathbf{F},\left[\begin{array}{cc}
A_{(k-1) \times(k-1)} & B_{(k-1) \times(n-k)} \\
& C_{(n-k) \times(n-k)}
\end{array}\right] \in P_{n-1}(\mathbf{F})\right\} .
$$


Note that $\varphi\left(E_{k k}\right) \varphi\left(a E_{k k}\right)=\varphi\left(a E_{k k}\right) \varphi\left(E_{k k}\right)=a\left[\varphi\left(E_{k k}\right)\right]^{2}$. This, together with (9), implies that $\varphi(A) \varphi\left(E_{k k}\right)=\varphi\left(E_{k k}\right) \varphi(A)$ for every $A \in S$. Thus,

$$
T^{-1} \varphi(S) T \subset \Gamma_{n}(\Lambda)
$$

where $\Lambda=\left\{i: \varepsilon_{i}=1\right.$ in (8) $\}$ is a subset of $[1, n]$. Due to Lemma 2.4, we obtain

$$
\operatorname{dim} \operatorname{span}(\varphi(S))
$$

$$
=\operatorname{dim} \operatorname{span}\left(T^{-1} \varphi(S) T\right) \leq \operatorname{dim} \operatorname{span}\left(\Gamma_{n}(\Lambda)\right) \leq 2^{-1}\left(n^{2}-2 n r+n+2 r^{2}\right) .
$$

On the other hand, we have

$$
\operatorname{dim} \operatorname{span}(S)=\operatorname{dim} \operatorname{span}\left(P_{n-1}(\mathbf{F})\right)+1=2^{-1}\left(n^{2}-n\right)+1
$$

Since $\varphi$ is bijective, so dim $\operatorname{span}(S)=\operatorname{dim} \operatorname{span}(\varphi(S))$. This, together with (10) and (11), gives that

$$
r \leq 1 \text { or } r \geq n-1
$$

Clearly, $r \neq 0$ since $\varphi\left(E_{k k}\right) \neq 0$. For the conclusion, it suffices to show that $r \neq n$. Otherwise, $r=n$ and $\varphi\left(E_{k k}\right)=I_{n}$. If $k=1$, we take $A_{i}=E_{1 i}$, $i=1,2, \cdots, n$ and $A_{n+1}=\sum_{j=2}^{n} E_{j j}$; if $k=n$, we take $A_{i}=E_{i n}, i=$ $1,2, \cdots, n$ and $A_{n+1}=\sum_{j=1}^{n-1} E_{j j}$; if $1<k<n$, we take $A_{i}=E_{i i}+\sum_{s=k+1}^{n} E_{s s}$, $i \in[1, k-1], A_{k}=\sum_{s=k+1}^{n} E_{s s}, A_{t}=E_{k t}, t \in[k+1, n]$ and $A_{n+1}=E_{k k}$. In every case, it is easy to check that

$$
\operatorname{dim} \operatorname{span}\left(A_{1}, \cdots, A_{n+1}\right)=n+1 .
$$

Again, note that $\varphi\left(E_{k k}\right)=I_{n}$ and $E_{k k}+A_{i} \in P_{n}(\mathbf{F}), i \in[1, n+1]$. This, together with Lemma 2.1, gives that $\varphi\left(A_{i}\right) \in P_{n}(\mathbf{F})$ for all $i \in[1, n+1]$. We will conclude that

$$
\varphi\left(A_{i}\right) \varphi\left(A_{j}\right)=\varphi\left(A_{j}\right) \varphi\left(A_{i}\right)
$$

for every part $i, j \in[1, n+1]$. When $k=1$, we have $P_{n}(\mathbf{F}) \ni \varphi\left(E_{11}+\left(A_{i}+\right.\right.$ $\left.\left.A_{j}\right)\right)=I_{n}+\varphi\left(A_{i}\right)+\varphi\left(A_{j}\right)$ for every $i, j \in[1, n]$. So, we have $\varphi\left(A_{i}\right)+\varphi\left(A_{j}\right) \in$ $P_{n}(\mathbf{F})$. Due to Lemma 2.1, proving (13) for $i, j \in[1, n]$. But $A_{i}+A_{n+1} \in P_{n}(\mathbf{F})$, for all $i \in[1, n]$, so we have by Lemma 2.1 that (13) holds for $i, j \in[1, n+1]$. 
The proof of (13) for the case $k=n$ is similar to the case $k=1$. Consider the case $1<k<n$. By the choice of $A_{1}, \cdots, A_{n+1}$, we see that (13) holds for $i, j \in[1, k] \cup\{n+1\}$ or $i \in[1, k] \cup\{n+1\}$ and $j \in[k+1, n]$. When $i, j \in[k+1, n]$, it follows by $P_{n}(\mathbf{F}) \ni \varphi\left(E_{k k}+\left(A_{i}+A_{j}\right)\right)=I_{n}+\varphi\left(A_{i}\right)+\varphi\left(A_{j}\right)$ that (13) holds. Hence, by Lemma 2.3 we have that $\varphi\left(A_{1}\right), \cdots, \varphi\left(A_{n+1}\right)$ can be simultaneously diagonalizable. It gives that $\operatorname{dim} \operatorname{span}\left(\varphi\left(A_{1}\right), \cdots, \varphi\left(A_{n+1}\right)\right) \leq$ $n$, which contradicts (12) since $\varphi$ is bijective. The proof is completed.

Lemma 3.2 Let $\varphi$ be a bijective linear preserver of idempotence on $T_{n}(\mathbf{F})$. Then there are an invertible matrix $T \in T_{n}(\mathbf{F})$ and a bijective map $g$ from $[1, n]$ to itself such that

$$
\varphi\left(E_{k k}\right)=T E_{g(k) g(k)} T^{-1}+\mu_{k k} I_{n} \text { for all } k \in[1, n]
$$

and

$$
\varphi\left(E_{i j}\right)=\left\{\begin{array}{l}
b_{i j} T\left(E_{g(i) g(j)}+D_{i j}\right) T^{-1}, g(i)<g(j), \\
b_{i j} T\left(E_{g(j) g(i)}+D_{i j}\right) T^{-1}, g(i)>g(j)
\end{array} \quad \text { for all } i<j \in[1, n]\right.
$$

where $\mu_{k k} \in\{0,1\}, b_{i j} \in \mathbf{F}^{*}, D_{i j}=\operatorname{diag}\left(\varepsilon_{1}^{(i j)}, \varepsilon_{2}^{(i j)}, \cdots, \varepsilon_{n}^{(i j)}\right)$ with $\varepsilon_{g(i)}^{(i j)}=\varepsilon_{g(j)}^{(i j)}$ for all $k, i, j \in[1, n]$, satisfying $(a)$ if char $\mathbf{F} \neq 2$ then $\mu_{k k}=0$; (b) if $\mathbf{F} \neq \mathbf{F}_{2}$ then $D_{i j}=0$.

Proof. For any $i \neq j$, since $E_{i i}, E_{j j}, E_{i i}+E_{j j} \in P_{n}(\mathbf{F})$, so $\varphi\left(E_{i i}\right), \varphi\left(E_{j j}\right), \varphi\left(E_{i i}\right)+$ $\varphi\left(E_{j j}\right) \in P_{n}(\mathbf{F})$. This, together with Lemma 2.1, implies that for any $i \neq j$, $\varphi\left(E_{i i}\right) \varphi\left(E_{j j}\right)=\varphi\left(E_{j j}\right) \varphi\left(E_{i i}\right)$ and in addition

$$
\varphi\left(E_{i i}\right) \varphi\left(E_{j j}\right)=0 \text { if } \operatorname{char} \mathbf{F} \neq 2 .
$$

Due to Lemma $2.3, \varphi\left(E_{11}\right), \varphi\left(E_{22}\right), \cdots, \varphi\left(E_{n n}\right)$ can be simultaneously diagonalizable by an invertible matrix $T \in T_{n}(\mathbf{F})$. Note that Lemma 3.1, so we may find a map $g$ from $[1, n]$ to itself such that $\varphi\left(E_{k k}\right), k \in[1, n]$ can be written in the forms

$$
\varphi\left(E_{k k}\right)=T E_{g(k) g(k)} T^{-1}+\mu_{k k} I_{n}, \text { for all } k \in[1, n],
$$

where $\mu_{k k} \in\{0,1\}$. 
If $\operatorname{char} \mathbf{F} \neq 2$, one can by (14) obtain that $\mu_{k k}=0$ and $g$ is bijective. If $\operatorname{char} \mathbf{F}=2$, we will show that $g$ is bijective, too. When $n=1$ or 2 , we have immediately that $g$ is bijective. Assume that $n \geq 3$. In order to prove this, we will conclude that if $g(i)=g(j)$ then $i=j$ for any $i, j \in[1, n]$. When $\mu_{i i}=\mu_{j j}$, then $\varphi\left(E_{i i}\right)=\varphi\left(E_{j j}\right)$ and so $i=j$ since $\varphi$ is a bijection. Next suppose that $\mu_{i i} \neq \mu_{j j}$. If $i \neq j$, we will get a contradiction. It is no loss generality to assume that $i<j, \mu_{i i}=0$ and $\mu_{j j}=1$. Take

$$
A_{k}= \begin{cases}E_{k k}+\sum_{s=i+1}^{j-1} E_{s s}+\sum_{s=j+1}^{n} E_{s s}, & k \in[1, i-1], \\ E_{i k}, & k \in[i, j-1] \cup[j+1, n], \\ E_{i i}+E_{j j}, & k=j, \\ \sum_{s=i+1}^{j-1} E_{s s}+\sum_{s=j+1}^{n} E_{s s}, & k=n+1 .\end{cases}
$$

It is easy to check that

$$
\operatorname{dim} \operatorname{span}\left(A_{1}, \cdots, A_{n+1}\right)=n+1 .
$$

Since $\varphi\left(E_{i i}\right)+\varphi\left(E_{j j}\right)=I_{n}$ and $\varphi\left(E_{i i}+E_{j j}+E_{i k}\right) \in P_{n}(\mathbf{F})$ for all $k \in$ $[i, j-1] \cup[j+1, n]$, we obtain that $\varphi\left(A_{k}\right) \in P_{n}(\mathbf{F})$ for all $k \in[1, n+1]$. Again, for any $s \neq t$, it is easy to see that $\varphi\left(A_{s}+A_{t}\right) \in P_{n}(\mathbf{F})$. This, together with Lemma 2.1 , gives that $\varphi\left(A_{s}\right) \varphi\left(A_{t}\right)=\varphi\left(A_{t}\right) \varphi\left(A_{s}\right)$ for any $s, t \in[1, n+1]$. Further, we see by Lemma 2.3 that $\varphi\left(A_{1}\right), \cdots, \varphi\left(A_{n+1}\right)$ can be simultaneously diagonalizable. Thus, $\operatorname{dim} \operatorname{span}\left(\varphi\left(A_{1}\right), \cdots, \varphi\left(A_{n+1}\right)\right) \leq n$. This contradict (16) since $\varphi$ is bijective. In every case, we have shown that $g$ is bijective.

For $i<j$, let us consider the image of $E_{i j}$ under $\varphi$.

When $\mathbf{F} \neq \mathbf{F}_{2}$, take $\mu \in \mathbf{F}$ with $\mu \neq 0,1$. Because of $\varphi\left(E_{i i}\right)+a \varphi\left(E_{i j}\right), \varphi\left(E_{j j}\right)+$ $a \varphi\left(E_{i j}\right) \in P_{n}(\mathbf{F})$ where $a=1, \mu$, we have by $(15)$ that $\left[\varphi\left(E_{i j}\right)\right]^{2}=0$ and

$$
\begin{aligned}
\varphi\left(E_{i j}\right) & =T E_{g(i) g(i)} T^{-1} \varphi\left(E_{i j}\right)+\varphi\left(E_{i j}\right) T E_{g(i) g(i)} T^{-1} \\
& =T E_{g(j) g(j)} T^{-1} \varphi\left(E_{i j}\right)+\varphi\left(E_{i j}\right) T E_{g(j) g(j)} T^{-1}
\end{aligned}
$$

By the direct computation, we get

$$
\varphi\left(E_{i j}\right)=\left\{\begin{array}{l}
b_{i j} T E_{g(i) g(j)} T^{-1}, g(i)<g(j), \\
b_{i j} T E_{g(j) g(i)} T^{-1}, g(i)>g(j),
\end{array} \quad \text { for all } i<j \in[1, n]\right.
$$

where $b_{i j} \in \mathbf{F}^{*}$. 
When $\mathbf{F}=\mathbf{F}_{2}$. Note that $\varphi\left(E_{i i}\right)+\varphi\left(E_{i j}\right), \varphi\left(E_{j j}\right)+\varphi\left(E_{i j}\right), \varphi\left(E_{j j}+E_{k k}\right)+$ $\varphi\left(E_{i j}\right) \in P_{n}(\mathbf{F})$ for all $k \neq i, j \in[1, n]$. It is easy to check that

$$
\left\{\begin{array}{c}
\varphi\left(E_{i j}\right)+\left[\varphi\left(E_{i j}\right)\right]^{2}=\varphi\left(E_{i i}\right) \varphi\left(E_{i j}\right)+\varphi\left(E_{i j}\right) \varphi\left(E_{i i}\right), \\
\varphi\left(E_{i j}\right)+\left[\varphi\left(E_{i j}\right)\right]^{2}=\varphi\left(E_{j j}\right) \varphi\left(E_{i j}\right)+\varphi\left(E_{i j}\right) \varphi\left(E_{j j}\right), \\
\varphi\left(E_{i j}\right)+\left[\varphi\left(E_{i j}\right)\right]^{2}=\left(\varphi\left(E_{j j}\right)+\varphi\left(E_{k k}\right)\right) \varphi\left(E_{i j}\right)+\varphi\left(E_{i j}\right)\left(\varphi\left(E_{j j}\right)+\varphi\left(E_{k k}\right)\right) .
\end{array}\right.
$$

Moreover, we get

$$
\left\{\begin{array}{c}
\left(\varphi\left(E_{i i}\right)+\varphi\left(E_{j j}\right)\right) \varphi\left(E_{i j}\right)=\varphi\left(E_{i j}\right)\left(\varphi\left(E_{i i}\right)+\varphi\left(E_{j j}\right)\right), \\
\varphi\left(E_{k k}\right) \varphi\left(E_{i j}\right)=\varphi\left(E_{i j}\right) \varphi\left(E_{k k}\right), \text { for all } k \neq i, j .
\end{array}\right.
$$

Note that $g$ is bijective, then it follows by (17) that

$$
\varphi\left(E_{i j}\right)=\left\{\begin{array}{l}
T\left(E_{g(i) g(j)}+D_{i j}\right) T^{-1}, g(i)<g(j), \\
T\left(E_{g(j) g(i)}+D_{i j}\right) T^{-1}, g(i)>g(j),
\end{array} \quad \text { for all } i<j \in[1, n],\right.
$$

where $D_{i j}=\operatorname{diag}\left(\varepsilon_{1}^{(i j)}, \varepsilon_{2}^{(i j)}, \cdots, \varepsilon_{n}^{(i j)}\right)$. Also, we have by $\varphi\left(E_{i i}\right)+\varphi\left(E_{i j}\right), \varphi\left(E_{j j}\right)+$ $\varphi\left(E_{i j}\right) \in P_{n}(\mathbf{F})$ that $\varepsilon_{g(i)}^{(i j)}=\varepsilon_{g(j)}^{(i j)}$. The proof is completed.

Now we can prove the main result of this paper.

Proof of Theorem 1.1. The "if" part. If $\operatorname{char} \mathbf{F} \neq 2$, the result is obvious. We assume that $\operatorname{char} \mathbf{F}=2$. We only prove it for $\varphi$ is of the form (1), since the proof when $\varphi$ is of the form (2) is similar. For any $A=\left[a_{i j}\right] \in$ $P_{n}(\mathbf{F})$, we will show that $f(A) \in\{0,1\}$. The conclusion is clear if $\mathbf{F}=\mathbf{F}_{2}$. Otherwise, $\mathbf{F} \neq \mathbf{F}_{2}$. Since $A^{2}=A$, so that $a_{k k}=0$ or 1 for all $k \in[1, n]$. Note that $f\left(E_{k k}\right) \in\{0,1\}$ and $f\left(E_{i j}\right)=0$ for all $k$ and $i<j$, then $f(A)=$ $\sum_{k=1}^{n} a_{k k} f\left(E_{k k}\right) \in\{0,1\}$. As $f(A) \in\{0,1\}$, it is easy to check that $\varphi(A)=$ $T A T^{-1}+f(A) I_{n}$ is idempotent. To continue, let us show that $\varphi$ is bijective. One way to prove this conclusion is to show that $\varphi\left(P_{n}(\mathbf{F})\right)=P_{n}(\mathbf{F})$ since $\operatorname{span}\left(P_{n}(\mathbf{F})\right)=T_{n}(\mathbf{F})$. Let $A \in P_{n}(\mathbf{F})$, then $T^{-1} A T \in P_{n}(\mathbf{F})$. We by above have known $f\left(T^{-1} A T\right) \in\{0,1\}$. When $f\left(T^{-1} A T\right)=0$, we have $\varphi\left(T^{-1} A T\right)=$ $A$; when $f\left(T^{-1} A T\right)=1$, by $f\left(I_{n}\right)=0$ we have $\varphi\left(T^{-1}\left(I_{n}+A\right) T\right)=I_{n}+A+$ $f\left(I_{n}+T^{-1} A T\right) I_{n}=A$. This completes the proof of the "if" part.

The "only if" part. It is known that $\varphi$ has the form in Lemma 3.2.

Firstly, let us prove that $D_{i j}=\mu_{i j} I_{n}$ where $\mu_{i j} \in\{0,1\}$ for any $i<j \in$ $[1, n]$. But from Lemma 3.2 we know that if $\mathbf{F} \neq \mathbf{F}_{2}$ then $D_{i j}=0$, so that we 
only need consider the case $\mathbf{F}=\mathbf{F}_{2}$. The case $n=2$ is obvious, so we assume that $n \geq 3$. We prove it only for $g(i)<g(j)$, since the proof for $g(i)>g(j)$ is very similar. Let $k \neq i, j$ be any integer in $[1, n]$. The proof is divided into the following four cases.

Case 1. $k<i$ and $g(k)<g(j)$. It follows from $E_{j j}+E_{k j}+E_{i j} \in P_{n}(\mathbf{F})$ that

$$
E_{g(j) g(j)}+E_{g(k) g(j)}+D_{k j}+E_{g(i) g(j)}+D_{i j} \in P_{n}(\mathbf{F})
$$

This tells us that

$$
\varepsilon_{g(k)}^{(k j)}=\varepsilon_{g(k)}^{(i j)}+1, \varepsilon_{g(i)}^{(k j)}=\varepsilon_{g(i)}^{(i j)}+1, \varepsilon_{g(j)}^{(k j)}=\varepsilon_{g(j)}^{(i j)}+1
$$

or

$$
\varepsilon_{g(k)}^{(k j)}=\varepsilon_{g(k)}^{(i j)}, \quad \varepsilon_{g(i)}^{(k j)}=\varepsilon_{g(i)}^{(i j)}, \varepsilon_{g(j)}^{(k j)}=\varepsilon_{g(j)}^{(i j)} .
$$

Note that $\varepsilon_{g(k)}^{(k j)}=\varepsilon_{g(j)}^{(k j)}$, so we see that $\varepsilon_{g(k)}^{(i j)}=\varepsilon_{g(j)}^{(i j)}$.

Case 2. $k<i$ and $g(j)<g(k)$. By $E_{j j}+E_{k j}+E_{i j} \in P_{n}(\mathbf{F})$, we get

$$
E_{g(j) g(j)}+E_{g(j) g(k)}+D_{k j}+E_{g(i) g(j)}+D_{i j} \in P_{n}(\mathbf{F}),
$$

this is impossible for any diagonal matrices $D_{i j}$ and $D_{k j}$. So the case dos not appear.

Case 3. $i<k$ and $g(i)<g(k)$. Similar to the proof of Case 1 , we have by $E_{i i}+E_{i k}+E_{i j} \in P_{n}(\mathbf{F})$ that $\varepsilon_{g(k)}^{(i j)}=\varepsilon_{g(i)}^{(i j)}$.

Case 4. $i<k$ and $g(k)<g(i)$. Similar to the proof of Case 2, we get a contradiction by $E_{i i}+E_{i k}+E_{i j} \in P_{n}(\mathbf{F})$.

The above Cases $1-4$ with $\varepsilon_{g(i)}^{(i j)}=\varepsilon_{g(j)}^{(i j)}$ deduce that $D_{i j}=\mu_{i j} I_{n}$ where $\mu_{i j}=\varepsilon_{g(j)}^{(i j)} \in \mathbf{F}_{2}=\{0,1\}$. So, we have that

$$
\varphi\left(E_{i j}\right)=\left\{\begin{array}{l}
b_{i j} T\left(E_{g(i) g(j)}\right) T^{-1}+\mu_{i j} I_{n}, g(i)<g(j), \\
b_{i j} T\left(E_{g(j) g(i)}\right) T^{-1}+\mu_{i j} I_{n}, g(i)>g(j),
\end{array} \quad \text { for all } i<j \in[1, n]\right.
$$

where $\mu_{i j} \in\{0,1\}$, and if $\mathbf{F} \neq \mathbf{F}_{2}$ then $\mu_{i j}=0$ for all $i<j \in[1, n]$. Now, by Lemma 3.2, similar to the proof of Theorem 3.1 in [11], we have $g(k)=k$ for all $k \in[1, n]$ or $g(k)=n-k+1$ for all $k \in[1, n]$. 
Suppose $g(k)=k$ for all $k \in[1, n]$ holds. If $\mathbf{F} \neq \mathbf{F}_{2}$, it follows from $\varphi\left(E_{i k}+E_{k k}+E_{k j}+E_{i j}\right) \in P_{n}(\mathbf{F})$ that $b_{i j}=b_{i k} b_{k j}$ for all $i<k<j \in[1, n]$. Set

$$
T_{1}=\operatorname{Tdiag}\left(1, b_{12}, \cdots, b_{1 n}\right) .
$$

We have by direct computation for any field that

$$
\varphi\left(E_{i j}\right)=T_{1} E_{i j} T_{1}^{-1}+\mu_{i j} I_{n} \text { for all } i \leq j \in[1, n],
$$

where $\mu_{i j} \in\{0,1\}$ satisfying $(a)$ if $\operatorname{char} \mathbf{F} \neq 2$ then $\mu_{i j}=0$ for all $i \leq j \in[1, n]$; (b) if $\mathbf{F} \neq \mathbf{F}_{2}$ then $\mu_{i j}=0$ for all $i<j \in[1, n]$. Now for any $A=\left[a_{i j}\right] \in T_{n}(\mathbf{F})$, let

$$
f(A)=\left(\sum_{i=1}^{n} \sum_{j=1}^{n} a_{i j} \mu_{i j}\right) I_{n},
$$

one can easy to check that $f$ is a linear map from $T_{n}(\mathbf{F})$ to $\mathbf{F}$. By (18) we get

$$
\varphi(A)=T_{1} A T_{1}^{-1}+f(A) I_{n} \text { for all } A \in T_{n}(\mathbf{F}) .
$$

From the definition of $f$ and the conditions of (18), we have $(a)$ if $\operatorname{char} \mathbf{F} \neq 2$ then $f=0 ;(b)$ if $\mathbf{F} \neq \mathbf{F}_{2}$, then $f\left(E_{i j}\right)=0$ for all $i<j \in[1, n]$. Again, for any $k \in[1, n]$, since $f\left(E_{k k}\right)=\left[b_{i j}\right] \in P_{n}(\mathbf{F})$ so that $b_{t t} \in\{0,1\}$ for all $t \in[1, n]$. When $\operatorname{char} \mathbf{F}=2$, we see by $\mu_{i j} \in\{0,1\}$ and the definition of $f$ that $f\left(E_{k k}\right) \in\{0,1\}$ for all $k \in[1, n]$. Note that $f\left(I_{n}\right)=f\left(\sum_{i=1}^{n} E_{i i}\right) \in\{0,1\}$ and $\varphi\left(I_{n}\right)=I_{n}+f\left(I_{n}\right) I_{n} \neq 0$. We get $f\left(I_{n}\right) \neq 1$ so that $f\left(I_{n}\right)=0$. The above all tell us that $\varphi$ is of the form (1). Similarly, if $g(k)=n-k+1$ for all $k \in[1, n]$ holds, we can prove that $\varphi$ is of the form (2). The proof is completed.

Proof of Theorem 1.2 . In order to prove the result, by Theorem 1.1 we only need to prove the following:

$$
\varphi\left(P_{n}(\mathbf{F})\right)=P_{n}(\mathbf{F}) \Longleftrightarrow \varphi \text { is bijective and } \varphi\left(P_{n}(\mathbf{F})\right) \subset P_{n}(\mathbf{F}) .
$$

The "£" part. By Theorem 1.1, $\varphi$ is of the forms (1) or (2). If $\operatorname{char} \mathbf{F} \neq 2$, the conclusion is clear. On the other hand, for $\operatorname{char} \mathbf{F}=2$, we already show that $\varphi\left(P_{n}(\mathbf{F})\right)=P_{n}(\mathbf{F})$ in the proof of the "if" part of Theorem 1.1.

The “ב" part. We only need to prove that $\varphi$ is bijective. In fact, by the following

$$
T_{n}(\mathbf{F})=\operatorname{span}\left(P_{n}(\mathbf{F})\right)=\operatorname{span}\left(\varphi\left(P_{n}(\mathbf{F})\right)\right) \subset \operatorname{Im} \varphi \subset T_{n}(\mathbf{F})
$$

we get $\operatorname{Im} \varphi=T_{n}(\mathbf{F})$. So $\varphi$ is surjective and furthermore it is bijective. 


\section{Acknowledgements}

This work was supported in part by the Chinese NSFs under Grant No. 10671026 and No. 10671033, the Fund of Heilongjiang Education Committee under Grant No. 1055G033.

\section{References}

[1] L.B. Beasley and N.L. Pullman, Linear operators preserving idempotent matrices over fields. Linear Algebra Appl., 146 (1991), 7-20.

[2] C.G. Cao and X. Zhang, Additive operators preserving idempotent matrices over fields and applications, Linear Algebra Appl. 248 (1996), 327-338.

[3] W.L. Chooi and M.H. Lim, Linear preservers on triangular matrices. Linear Algebra Appl., 269(1998), 241-255.

[4] A. Fošner, Automorphisms of the poset of upper triangular idempotent matrices, Linear and Multilinear Algebra, 53(2005)27-44.

[5] A. Guterman, C.K. Li and P. Šemrl, Some general techniques on linear preserver problems. Linear Algebra Appl., 315 (2000), 61-81.

[6] S. Jondrup, Automorphisms and derivations of upper triangular matrix rings, Linear Algebra Appl. 221 (1995) 205-218.

[7] C.K. Li and S. Pierce, Linear preserver problems. Amer. Math. Month., 108 (2001), 591-605.

[8] S.W. Liu, Linear maps preserving idempotence on matrix modules over principal ideal domains, Linear Algebra Appl. 258 (1997), 219-231.

[9] S.W. Liu and D.B. Zhao, Introduction to linear preserver problems. Harbin press, 1997, Harbin.

[10] L. Molnar and P. Šemrl, Some linear preserver problems on upper triangular matrices, Linear and Multilinear Algebra, 45(1998),189-206. 
[11] X.M Tang, C.G. Cao and X. Zhang, Modular automorphisms preserving idempotence and Jordan isomorphisms of triangular matrices over commutative rings, Linear Algebra Appl., 338 (2001), 145-152.

[12] X. Zhang, Idempotence-preserving linear operators on triagular matrices over commutative integral ring (in Chinise), J. Xinjiang Univ. Natur. Sci, 10(2)(1992): 25-27.

[13] X. Zhang and C.G. Cao, Homomorphisms between additive matrix groups which preserve some invariants (in Chinese). Harbin Press, 2001, Harbin.

Received: December 29, 2006 